\title{
Fenomenología y psicoterapia humanista-existencial
}

\author{
Phenomenology and \\ humanistic-existencial psychotherapy
}

\author{
*AndréSassenfeld J onquera, \\ ${ }^{*}$ Laura M oncada Arroyo
}

\section{Resumen}

En este artículo se presenta como marco referencial para la psicoterapia humanista-existencial, al gunos conceptos fundamentales dela fenomenología y el método fenomenológico que iluminan el quehacer psicoterapéutico. Con la finalidad de dar cumplimiento a este objetivo, en primer lugar, se delinea la manera en la que los mismos psicoterapeutas humanistasexistenciales entienden el núcleo de la aproximación fenomenológica y, en segundo lugar, se especifica al gunos usos posibles de la fenomenología en el área de la teoría y la práctica clínica.

Palabras clave: Fenomenología, psicoterapia humanista-existencial, práctica clínica.

\section{Abstract}

This article presents some fundamental concepts of phenomenology and the phenomenological method that throw somelight on psychotherapeutic practice as theoretical frame for humanistic-existential psychotherapy. To accomplish this, first the way in which humanistic-existential psychotherapists understand the nucleus of the phenomenological approach is outlined and second some possible uses of phenomenology in the area of clinical theory and practice are specified.

Key words: Phenomenology, humanistic-existential psychotherapy, clinical practice.

* Psicólogo. Equipo Clínico H umanista, D epartamento de Psicología, Universidad de Chile. Email: asjorquera@hotmail.com

** Psicóloga. Profesor Asistente, D epartamento de Psicología, Equipo Clínico H umanista, Universidad de Chile. Email: equipohumanista@uchile.cl 


\section{Introducción}

Tradicional mentela psicoterapia de orientación humanista-existencial hasido asociada a una fundamentación filosófica y metodológica decarácter fenomenológico (G endlin, 1966; K riz, 1985; M ay, 1961; M oreira, 2001; M oreira, Saboia, Beco \& Soares, 1995; M oss, 2001; Q uitman, 1985; Tageson, 1982; Yontef, 1993, 1998, 2004). Sin embargo, el conocimiento teórico y práctico que los psicoterapeutas de orientación humanista-existencial tienen, por un lado, dela fenomenología y susdesarrollos conceptuales, y por otro, de las posibilidades y losimperativos dela aplicación clínica del método fenomenológico, a menudo es vago einexacto. Por lo tanto, este conocimiento, si efectivamenteconsideramosquela filosofía fenomenológicaesuno de losfundamentos dela psicología humanista-existencial (como haremos en el contexto deesteartículo), tiendeaser insuficientey puede, eventualmente, deformar y limitar $\mathrm{l}$ ejercicio profesional deuna psicoterapia humanista-existencial orientada en profundidad por sus propios planteamientos cardinales.

En la actualidad, terapeutas de diversas orientaciones han comenzado a reconocer y subrayar la relevancia de los supuestos filosóficos que subyacen a la práctica psicoterapéutica y han detallado al gunas de sus múltiplesimplicancias concretas. Así por ejemplo, la natural eza de la relación terapéutica, la comprensión del impasseo la conceptualización de la psicopatología (A. Goldberg, 2001; C. Goldberg, 2000; M oreira, 2001; M oreira et al., 1995; O range, Atwood \& Stolorow, 1997; Spagnuolo, 2001; Stolorow, Atwood \& O range, 2002; Stolorow, O range \& Atwood, 1998; Velasco, 2003; Wolf, 1988).

En el plano delaformulación teórica dela psicoterapia humanista-existencial, esteénfasis en la explicitación delosfundamentosfilosóficosseremontaal menoshasta las contribuciones tempranas de Frankl (1946) y de M ay (1961) y ha sido, desde entonces, una preocupación recurrentedelosrepresentantes deesta aproximación. A la luz detal es desarrollos, queda demanifiesto la importancia decisiva quetieneel grado deconocimiento quelos psicoterapeutas poseen respecto de las suposicionesfilosóficas quefundamentan su forma deacercarse al trabajo terapéutico.

En el contexto esbozado, esteartículo intenta describir y destacar uno delos pilares capital es de la psicoterapia de orientación humanista-existencial, cuyo conocimiento es esencial para todo terapeuta que trabaja o desea trabajar siguiendo losprincipios definitorios deesteacercamiento -la fenomenología y el método fenomenológico. M ásquecentrarsetan sólo en los aspectos propiamentefilosóficosdeestatemática, se pretendeabordarla desdeuna perspectiva tanto conceptual como aplicada. Con la finalidad de dar cumplimiento a esteobjetivo, en primer lugar se delinea la manera en la quelosmismospsicoterapeutas humanistas-existenciales entienden el núcleo dela aproximación fenomenológicay, en segundo lugar, se especifican al gunos usos posibles 
dela fenomenología en el área dela teoría y de la práctica clínica.

\section{Algunas consideraciones generales sobre la fenomenología}

Lafenomenología como sistemafilosófico coherentetienesusorígenesformales en la obra deEdmund $\mathrm{H}$ usserl (1859-1938). A pesar deesto, el término fenomenología fue utilizado con anterioridad por filósofostan importantes como $\mathrm{H}$ egel (recuérdese su tratado capital Fenomenología del espíritu [1807]) y algunas de sus nociones conceptualesfundamental es están ya presentes en las reflexiones de Brentano y otros pensadores. D esdehace mucho tiempo, sin embargo, la fenomenología es entendida más como un movimiento filosófico amplio que reúne diversos desarrollosheterogéneosen una mismaárea que como un sistema teórico estrechamentedelimitado por los planteamientos del mismo H usserl (M oreira, 2001; M oreno, 2000a, 2000b; Waldenfels, 1992). La afinidad básica de estos diferentes desarrollos no debe buscarse en el ámbito de contenidosy concepciones específicas, sino más bien en la aproximación metodológica particular que orienta la investigación fenomenológica en general. C on todo, el trabajo deH usserl constituyeel punto de partida indispensablepara cualquier estudioso que desee adentrarse, demodo informado y sistemático, en el acercamiento fenomenológico.

H usserl estabainsatisfecho con los resultados arrojados por lasaplicaciones del método científico positivista en el campo del estudio del mundo externo y la realidad psicológica. D esdesu perspectiva, la imagen del mundo y del ser humano quela ciencia estabaformándoseen el transcurso del siglo XIX y lasprimeras décadas del siglo XX se alejaba excesivamente dela experiencia genuina quelas personastienen de su entorno y desí mismas y, en este sentido, no podía ser visualizada como empírica y fidedigna. En esta línea, en una contribución temprana, Gendlin (1966) indica que H usserl rechazó la teoría científica-difundida hasta el día de hoy- según la cual vemos "perceptos" o pensamos "imágenes" al advertir que, en su experiencia personal directa einmediata, nunca había visto un percepto o pensado unaimagen. M ásbien, razonó H usserl, losprocesospsíquicossiempreson intencionales-es decir, invariablementehacen referencia y están ligados a objetosy/ o sucesos que se encuentran en la realidad externa (H alling \& Carroll, 1999; Waldenfels, 1992). Por lo tanto, entenderloscomo sencillas ocurrencias subjetivas que no guardan relación con el mundo en el cual las personas existen y se desenvuelven es una abstracción excesiva.

La intencional idad delas vivencias humanasimplica que siempreobservamos una casa, un árbol o al guna otra cosa existenteen el mundo y siemprenosenojamoso entristecemos por lo quealguien hizo o por algo que ocurrió. D esde esta perspectiva, 
parala fenomenología el individuo "nunca experimenta una 'experiencia' o unos sentimientos; siempretratan, se derivan o están en situaciones-en-el-mundo" (G endlin, 1973, p. 144). D eestemodo, lo que

\begin{abstract}
"experienciamos, de hecho, elimina la barrera entre lo objetivo (átomos concebidos geométricamente y fuerzas físicas externas) y lo subjetivo (fuerzas y entidades internas). H usserl encontró que el mundo humano total realmente estaba implícito en nuestra experiencia y que las supuestas entidades internas eran meros constructos teóricos" (G endlin, 1966, p. 42).
\end{abstract}

Aś, lafenomenología representa un intento sostenido por superar o trascender la dicotomización categórica entre sujeto y objeto, queesinherenteal desarrollo del pensamiento occidental (H alling \& C arroll, 1999; Q uitmann, 1985; Yontef, 1998, 2004). Las experiencias, tal como el individuo las vivencia, no son separables de y siempreestán dirigidas hacia algo, son sobre algo, de algo o con algo; constituyen una reacción a al go y nunca corresponden tan sólo a una especie de entidad concreta y aislada dentro de una menteo un cuerpo determinado.

H usserl consideraba quelaciencia desu época, en términos generales, secaracterizaba por un alto grado de ingenuidad. D ebido a la completa inmersión de las actividadescientíficasmismas en el mundo práctico einmediato delavida (Lebenswelt), laciencia incurriría en el graveerror deno poner en entredicho y de dar por supuesto, lo queH usserl Ilamaba "actitud natural" como hecho incuestionable que reflejaríala natural eza fundamental dela realidad -esto es, la creencia cotidiana en la efectividad de la separación entresujeto y objeto, queincluyela suposición deque existeunarealidad objetiva independiente dequien la percibey estudia (Brazier, 1993; $\mathrm{H}$ alling \& CarroII, 1999).

A la actitud natural, lafenomenología husserliana opone la actitud fenomenológica, al canzablea través de la aplicación disciplinada del método fenomenológico como fundamento para investigar y entender demanera exactay empírica la experiencia queel ser humano tiene del mundo, de sí mismo y de sus semejantes. La actitud fenomenológica posibilita, a quien la asume, suspender temporalmentela propia inserción congénita en el mundo einterrumpir con ello, el movimiento habitual haciala realidad exterior para poder contemplarlo sin participar en él (M oreira, 2001). En otras palabras, la actitud fenomenológica permite aprehender y observar la intencional idad delos procesos psicológicos como estructura constitucional y pre reflexiva dela experienciahumana.

Demodo similar aH usserl, lospioneros del movimiento humanista-existencial reconocieron quelas concepciones psicológicas más importantes elaboradas en la primera mitad del siglo XX -en particular, el psicoanálisis y el conductismo- sehabían alejado en gran medida de las experiencias efectivas de las personas y sehabían vuel to en exceso abstractas y estériles. Al menos en parte, los psicoterapeutas humanistas- 
existencial es encontraron en lafenomenología un fundamento filosófico y metodológico útil debido a la semejanza y afinidad con sus respectivos puntos de partida. A partir de estereconocimiento y, siguien do la conocida consigna programática de H usserl ("DD evueltaalascosasmismas!") ${ }^{1}$, desarrollaron psicologías cercanas a lo humanamente experimentableque sebasan en la experiencia directa einmediata (M 0ss, 1999; Q uitmann, 1985; Tageson, 1982). H usserl, antes que ellos, ya había insistido en numerosas ocasiones en el hecho de que todo cuanto sabemos del mundo y del ser humano lo conocemos mediantenuestra experiencia y, en consecuencia, que "el mundo debepercibirsedel modo en quese presenta a cadauno en el marco desus experiencias" (Q uitmann, 1985, p. 47) con la finalidad dealcanzar un entendimiento psicológico comprehensivo.

\section{Aspectos fundamentales del método fenomenológico}

Podemos afirmar que desde el punto de vista de la fenomenología, los dos hechos básicos que guían la investigación y que, dado el énfasis en el concepto de intencionalidad, seimplican uno al otro, son la experienciay el fenómeno. Un fenómeno es la apariencia o la forma específica en la queel objeto de estudio se presenta a sí mismo de modo inmediato a la consciencia del observador (M ay, 1969; Tageson, 1982). En tal sentido, la fenomenología suponequelosfenómenos-en cuanto constituyen uno de los dos aspectos centrales de la relación intencional entre individuo y mundo- son lo único queal ser humano lees accesibley cognoscibledirectamente. Por lo tanto, la concepción fenomenológicadelo real, tal como ha indicado insistentemente Yontef $(1993,1998,2004)$, es relacional, ya que asume que la realidad debe ser entendida como una co-construcción queseproduceentreel observador y lo observado. Así, surge la necesidad de reconocer la existencia de innumerables realidades válidas. Por otro lado, lo real siempresenos presenta como un tejido sólido que está dado deantemano y en el cual nosencontramos. D ebido a esto, la realidad fenoménica esun a priori en nuestra experiencia (M oreira, 2001).

El otro aspecto complementario dela relación intencional queuneal observador y a lo observado, es la experiencia, es decir, nuestra forma personal de experimentar el hecho denuestra existenciaen el mundo y delosfenómenosinternosy externos de momento amomento. Puesto que H usserl pensaba quela experienciainmediata es 10 único que podemos llegar a conocer con certeza, estaba interesado en entenderlasién-

\footnotetext{
${ }^{1}$ H usserl declaraba: "Regreso del discurso de las cosas, a las cosas mismas, tal y como aparecen en la realidad, a nivel de los hechos vividos, previos a cualquier elaboración conceptual deformante" (citado en Peñarrubia, 1998, p. 67).
} 
dole tan fiel como fuera posible. En consecuencia, desechó teorías y especulaciones abstractas y buscó quelos conceptos emergieran directamentedelasvivenciasinmediatas y concretas delas personas (Gendlin, 1973; Tageson, 1982). D eseabaalcanzar una comprensión exacta del ámbito dela experienciaqueexistecon anterioridad al mundo abstracto descrito por la ciencia, el mundo dela vida (Lebenswelt) tal como es experimentado, en efecto, por los individuos (H alling \& C arroll, 1999; Tageson, 1982). A hora bien, lo dicho no significa quelafenomenología, tal como a veces se ha sostenido, rechace la formulación deteorías que den cuenta delosfenómenosinvestigados. M ás bien, tal como señala M oreira (2001), la investigación fenomenológica busca permitir que la teoría emerja a partir del encuentro -que es, de hecho, una unión inseparable entrela experiencia y el fenómeno. La teoría naceen el entrelazamiento de la universalidad del conocimiento y lasingularidad de la vivencia del investigador, por lo quees, en consecuencia, inevitablemente relativa, inacabada y modificable.

Como hemos visto, fenómeno y experiencia representan los pilares que sostienen el método fenomenológico. También ha quedado al descubierto queel objetivo cardinal dela investigación fenomenológica es la comprensión delos fenómenos tal como se muestran a la consciencia de quien los experimenta y observa ${ }^{2}$. $\mathrm{H}$ alling y Carroll (1999) definen lafenomenología como "el estudio metodológicamenteriguroso y no sesgado de las cosas tal como aparecen, de manera que se pueda llegar a un entendimiento esencial dela consciencia humana y su relación con losobjetosdela experiencia” (p. 96). Ahorabien, en la tradición fenomenológica, la comprensión deun fenómeno es diferenciada con claridad de una explicación que dé cuenta de él; la fenomenología está dedicada a comprender la experiencia queel observador tienedel fenómeno en sus propiostérminosy asumeque esto sólo sepuede lograr mediantela descripción comprehensiva y detallada de ésta, rechazando el análisiscausal explicativo o losjuicios deval or como herramientasútiles para arribar aun entendimiento genuino del objeto de investigación involucrado (Brazier, 1993; Ginger \& Ginger, 1987; M oreira, 2001; Peñarrubia, 1998). Sesuponequeel quéy el cómo preceden necesariamenteal por qué. D iceKurt Lewin: “U na representación correctadeaquello que'es' es,

\footnotetext{
${ }^{2}$ Dicho sea de paso, la obra filosófica de H usserl puede ser dividida en distintas etapas. Algunos escritos tardíos de $\mathrm{H}$ usserl, en ocasiones clasificados como pertenecientes a la denominada fenomenología trascendental, incluyen entre otras cosas, reflexiones sobre la posibilidad de alcanzar un conocimiento completamente objetivo de los fenómenos investigados. En términos generales, los psicoterapeutas de orientación humanista-existencial no se adscriben y no emplean los conceptos de la fenomenología trascendental, sino aquellos característicos de una etapa anterior del pensamiento de H usserl, llamada fenomenología descriptiva (Yontef, 1998, 2004). Por otro lado, los psicólogos humanistas-existenciales se han centrado en lo que Yontef $(1998,2004)$ llama fenomenología psicológica, esto es, aquellas investigaciones fenomenológicas cuyos objetos de estudio han sido los procesos psíquicos y la existencia humana. Lo que resta de este artículo se basa en el método y los descubrimientos relacionados con esta última área de la fenomenología.
} 
al mismo tiempo, una 'explicación' deaquello que ocurre" (1969, citado en Soff, Ruh \& Zabransky, 2004, p. 25).

Así, el punto departida fundamental dela investigación fenomenológica es la descripción lo máscompleta y exhaustiva posibledel fenómeno a partir de la experiencia directa e inmediata que el investigador tiene de éste, con lo cual se garantiza la consideración de la singularidad eirrepetibilidad de cada fenómeno observado. Esto implica que los fenómenos han de estudiarse y describirse en concordancia con la noción deintencional idad, teniendo en cuenta tanto las circunstancias externas en las cuales semanifiestan como los procesos psíquicos relacionados de quien los estudia ${ }^{3}$ (Peñarrubia, 1998; Soff et al., 2004; Yontef, 1993). En este contexto, el fenómeno es entendido como aquello que es aparenteo "dado" en una situación o en un proceso subjetivo o intersubjetivo quees deinterés. Como señala Yontef (1993), la fenomenología intenta al canzar una comprensión basada en lo que es obvio o revelado por el objeto deestudio y no en los juicios del observador. Dicho deotro modo, laatención se Ileva a aquello que está presente y que es observable y no a aquello que uno puede sospechar como eficaz detrás delas apariencias.

A su vez, la exploración fenomenológicaapuntaa la descripción claray detallada delo queeso estásiendo y por lo tanto desincentiva, en el transcurso dela realización dela descripción del fenómeno, la inclusión detodo lo quefue, será, podría o debiera ser. La información que interesa proviene delo que se vivencia inmediata eingenuamenteen un momento determinado. En términos concretos, esto significaque, para la fenomenología psicológica, la herramienta esencial de investigación está dada por la vivencia del investigador o fenomenólogo tal como es percibida o sentida demanera corporal, afectiva e intelectual, en el aquí-y-ahora (Ginger \& Ginger, 1987; Yontef, 1993). Como veremos más adelante en relación a la psicoterapia, esta concepción puedeser aplicada tanto al psicoterapeuta como al cliente.

El hecho queel método fenomenológico sebasey secentreen la experienciadel investigador, introduce una dificultad importantequeno pasó desapercibida ni en la obra deH usserl ni en la desus sucesores: suponiendo quefuera necesario, las experienciasindividuales son difíciles-sino imposibles- dehomogeneizar y, en consecuencia, distintas descripciones fenomenológicas deun mismo fenómeno divergirán inevitablemente. Esta problemática nuclear, queinvolucra los sesgos que pueden determinar,

\footnotetext{
${ }^{3} \mathrm{G}$ endlin $(1966,1973)$ indica que uno de los grandes descubrimientos de $\mathrm{H}$ usserl fue su reconocimiento de quela experiencia sentida no es una mera entidad intrapsíquica, sino que contiene al menos implícitamente situaciones o aspectos del mundo de la vida cotidiana. A esta concepción se debe la importancia de incluir en la exploración fenomenológica tanto los datos fenoménicos como los datos experienciales. Como habíamos dicho, la idea del fenómeno se refiere a la apariencia en la que el objeto de estudio se presenta a la consciencia de quien lo investiga y la experiencia hace referencia a la forma en la que el investigador fenomenológico experimenta los fenómenos internos y externos.
} 
limitar y/o distorsionar la experiencia que un individuo específico tenga de un cierto fenómeno, ha sido abordadatanto desdeel punto de vista epistemológico como desde el metodológico:

(1) En el plano epistemológico, ya hemosafirmado que la fenomenología no se adscribeal ideal positivista deobjetividad y neutralidad, ni a la noción primaria deun mundo objetivo independiente de quien lo observa. Por el contrario, se suponela continua co-constitución y co-construcción dela realidad a partir de la interacción inevitable entre persona y mundo ( $\mathrm{H}$ alling \& C arroll, 1999; M artínez, 1982; Yontef, 1998, 2004). D ebido a ello, se definela realidad en términos dela acción intencional deun observador en relación a un mundo fenoménico. Esta esuna concepción que, como lo han puesto al descubierto Stolorow y suscolegas (2002), defiendela existenciade innumerablesmundos deexperiencia posiblescuyo valor último de realidad es relativo y dependiente devariablescontextuales.

(2) En el plano metodológico, la fenomenología reconoce la necesidad de restringir los sesgos dela experiencia del investigador tanto como sea posible, aunque se admita que esta restricción nunca puede ser del todo satisfecha. Como diceYontef (1993), debido a queel método fenomenológico sebasaen la experiencia, sevuelveimperativo diferenciar con claridad entre lo efectivamente vivenciado y todo el conjunto de elementos asumidos, establecidos, aprendidosy/o inferidosquedeterminan y sesgan la experiencia del fenómeno tal como se presenta. Así, desde una perspectiva operacional, un aspecto relevante del método fenomenológico es el proceso continuo y sistemático de refinamiento de la capacidad de darse cuenta del investigador y de reducción decualquier prejuicio o preconcepción quese alberguerespecto del fenómeno en cuestión ${ }^{4}$.

En el contexto esbozado, la "puesta entreparéntesis" de elementostalescomo losvalores personales, las suposiciones, las inferencias o los prejuicios metafísicos y los juiciossobrequéeslo relevanteen unasituación concreta a la hora de describir la propia experiencia deun fenómeno, esla manifestación paradigmática delo quecon anterio-

\footnotetext{
${ }^{4}$ Ya en sus primeros escritos, H usserl había insistido en la necesidad de aprender a despejar las preconcepciones que influencian y deforman la propia experiencia. Su afirmación de la "vuelta a las cosas mismas" es una expresión de ello (H alling \& Carroll, 1999). Su crítica a la denominada "actitud natural" y su consiguiente búsqueda de la comprensión de la experiencia pre-reflexiva del mundo dela vida, es otro ejemplo de esta concepción general, la que queda plasmada en la noción de epoché o "puesta entre paréntesis" como línea directriz metodológica.
} 
ridad Ilamamos la actitud fenomenológica. La práctica dela epoché, o suspensión temporal denuestras preconcepciones, exige disciplina y consciencia. Su finalidad central es, por un lado, ayudar al observador a distanciarse de su forma habitual de pensar y sentir para que sea capaz de advertir la crucial diferencia entre lo que efectivamente percibey experimenta en un momento dado y aquello quees un residuo del pasado y, por otro lado, permitir queel investigador vivenciecon lamenor contaminación posible lo que revela el fenómeno involucrado (Yontef, 1993). “La puesta entreparéntesis apoya al fenomenólogo (terapeuta) para que sea consciente delos sesgos acerca dequé es lo real, acerca de cuálesson los datosválidos y acerca de preconcepciones generales, y para que advierta suposiciones prematuras respecto del conocimiento de 'la verdad'" (Yontef, 1998, p. 5). Estepunto de vista despeja la mente del observador, posibilita el reconocimiento de las propias preconcepciones en cuanto tales y promueve una actitud de apertura a lo "dado" que nos vuelve permeables a la eventual e inesperada relevancia de datos que podrían haber sido desechados como irrelevantes o incluso ignorados del todo.

Siguiendo a M oreira (2001), la necesidad dela puesta entreparéntesissurgede lainserción constitucional del ser humano en el mundo delavida, un hecho existencial quehaceinevitablelaimplementación deesta especiedeartificio para poder trascender la actitud natural y llegar al fenómeno mismo -en este contexto, volver a las cosas mismas significa volver al mundo tal como senos presenta con anterioridad a nuestro conocimiento de él y a toda determinación científica, abstracta y simbólica de sus características. La epochéexigeal investigador la compleja y sistemática disciplina de agudizar su capacidad de consciencia con la finalidad de lograr suspender temporalmente sus conocimientos pasados, sus experiencias previas, sus diversas teorías, sus diferentes objetivosy cual quier otro elemento que pudiese sesgar su acercamiento al fenómeno estudiado (H alling \& C arroll, 1999; M oreira, 2001; Yontef, 1993, 1998, 2004).

El método fenomenológico secentra en la aceptación plenadetodos los datos fenoménicosy en la consecuente descripción deestostal como son experimentadosen el presente, con la menor cantidad posible de supuestos y transformaciones contaminantes dela percepción. D eacuerdo a Yontef (1998), desdeun inicio se abandona la pretensión de objetividad y neutralidad completas y se reemplaza este ideal por un intento disciplinado de ser nuevamente influenciado tanto como sea posible por las características fundamentales dela experiencia viva. 


\section{Fenomenología y psicoterapia humanista-existencial}

A lospsicoterapeutas deorientación humanista-existencial lesinteresa en particular la exploración fenomenológica delosfenómenos propiamentehumanosy psicológicos-una aproximación dedicada a la investigación de un objeto de estudio representado por la existencia y la experiencia conscientedel individuo, queYontef (1993, 1998, 2004) y M oreira (2001) denominan fenomenología existencial o psicológica. En el marco dela psicoterapia humanista-existencial, el método fenomenológico tiene unamultitud deusos posibles, delos cual es destacaremos al gunas aplicaciones específicasligadasala relación psicoterapéutica y a los procesos dinámicosquela caracterizan.

\section{La situación psicoterapéutica y la atención del psicoterapeuta}

D esdeel punto de vista fenomenológico, la situación terapéutica consisteen un contexto singular constituido por la interacción intersubjetiva continua deal menos dos fenomenologías distintas. Tanto el psicoterapeuta como el paciente(o cliente), en su calidad de sujetos, están inmersos en el inevitable flujo tanto de sus respectivas experiencias dela situación relacional como con respecto al otro duranteel transcurso decada sesión detrabajo psicoterapéutico. Brazier $(1992,1993)$ piensa quela atención fenomenológica del terapeuta puedecentrarseen distintos "camposfenoménicos" quelo conducirán hacia direcciones divergentes de intervención clínica. Para Brazier, estoscamposincluyen: los contenidos del mundo interno del clientetal como éstelos comunica con palabrasy otras manifestacionesintencionales; la percepción directaque el terapeuta tiene de la forma y cualidad concreta de las expresiones verbales y no verbales de la persona del cliente; la consciencia del terapeuta respecto de su propio mundo interno y del mundo compartido designificados quese desarrolla a lo largo del proceso psicoterapéutico entre los participantesinvolucrados. En estesentido, todo fenómeno que es explorado en psicoterapia, con independencia de si se trata de un proceso pśquico propio del pacienteo de un proceso vincular queinvolucraal cliente y al terapeuta o al clientey a al guna otra persona significativa para él, puede ser objeto deal menos dosdescripciones fenomenológicas distintas que, por lo común, tienden a enfatizar o abarcar aspectos dispares. 


\section{La fenomenología en algunas aproximaciones psicoterapéuticas de orientación humanista-existencial}

D iferentes aproximaciones psicoterapéuticas de orientación humanista-existencial han enfatizado al gunos deloscamposfenoménicos descritospor sobreotros. La psicoterapia centrada en la persona, formulada por R ogers $(1951,1961)$ y sus seguidores, al menos en susinicios, se basó en reflejar y con ello clarificar y transformar el mundo interior del clientetal como éstelo describeal psicoterapeuta. Rogers inicialmentesupuso queel terapeuta, con la final idad decomprender de manera empáticay cabal la realidad vivencial del cliente, debía prescindir dela utilización clínica desus propias descripcionesfenomenológicas. Sin embargo, con posterioridad llegó adesta car la importancia terapéutica de un encuentro existencial entre dos personas que comparten sus respectivos mundos experienciales-es decir, la relevancia deun enrique cimiento mutuo a partir de las características diferenciales delas fenomenologías propias del terapeutay del cliente.

Por otro lado, los psicoterapeutas corporales que siguieron y desarrollaron las teorías y técnicas creadas por Reich (1942, 1949), pasaron del énfasis reichiano original sobre la observación fenomenológica inmediata que el terapeuta hace del cuerpo del paciente como modal idad primaria que orienta las intervenciones clínicas, hacia una mayor consideración por los procesos psicológicosy corporalestal como son experimentadosy descritosfenomenológicamentepor el pacienteen la situación psicoterapéutica (Totton, 2003). D esdeesta perspectiva, la psicoterapia post-reichiana seha desarrollado en términostécnicoshaciael intento detransformar la experienciaqueel clientetienede sí mismo y desu propia realidad corporal, alejándosedel interés por intervenir demodo mecánico sobresu cuerpo con lafinalidad degenerar cambiosobservablesqueno siempre implican un correlato vivencial quesesostenga en el tiempo.

Losterapeutasgestálticos, por su parte, desdelos comienzosformales desu acercamiento a la obradePerls $(1947,1951)$, han puesto al descubierto la utilidad clínica de la contrastación y clarificación delas diferencias más llamativas entre las descripciones fenomenológicas delaexperiencia del clientey lashechas por su psicoterapeuta. También han articulado una metodología psicoterapéuticaquesecentra en la exploración vivencial dela experiencia inmediatadel paciente, posibilitando la ampliación desu capacidad de darsecuentarespecto defacetasno reconocidasdeésta. En la mayoría de las aproximacionespsicoterapéuticashumanista-existencial es, laaceptación general del empleo oportuno de la auto-revel ación del psicoterapeuta como herramienta clínica valiosa-al gunos de cuyos primeros partidariosfueron losterapeutas gestálticos- haintroducido su fenomenología como ingredienteesencial del diálogo terapéutico. 


\section{Fenomenología y experiencia en el contexto de la relación psicoterapéutica}

Con ciertaindependencia delos acentos específicos mencionados, las diversas formas de psicoterapia fundamentadas en el método fenomenológico, comparten un interés básico por las múltiples dimensiones dela experienciafenoménica presente del psicoterapeuta y del cliente, y por los diferentes significados que ambos les atribuyen (M oreira, 2001). En el contexto psicoterapéutico, la "vueltaa las cosas mismas" planteada por $\mathrm{H}$ usserl, seconvierteen una vuelta a las vivencias actuales en su estado bruto, directo einmediato. Entreotras implicancias, esto significa, siguiendo a Brazier (1993), que el terapeuta da inicio a un proceso terapéutico en general y a cada sesión en particular, desdeel lugar experiencial y existencial en el cual seencuentra el pacienteen un momento determinado -a lo cual podríamos agregar, también, el lugar experiencial y existencial en el cual seencuentra el mismo psicoterapeuta en un momento dado. D esdeesta perspectiva, la epochécomo intento de adentrarseen un estado degenuina apertura a la inmediatez de la experiencia propia y dela del otro, sevuel veuna herramienta imprescindiblepara guiar el trabajo psicoterapéutico:

“Las posibilidades de crecimiento psicológico de un individuo están en
proporción [directa] a la medida en la que las presuposiciones pueden ser
puestas entre paréntesis. [... ] Como terapeutas, tenemos que examinar
nuestro trabajo una y otra vez con el objeto de reconocer qué es lo que
damos por supuesto, qué presuposiciones estamos haciendo. Esto no es
fácil. Es la tentativa de ver lo que no estamos viendo" (Brazier, 1993, p. 2).

\section{La epoché como actitud fundamental del psicoterapeuta}

La tarea fenomenológica principal del psicoterapeuta consisteen aprender a poner progresivamente entre paréntesis las distintas presuposiciones queal berga respecto del cliente, incluyendo en especial su tendencia a escuchar y prestar atención a ciertas cosas y no a otras, tendencia queguardaíntima relación con lasteoríaspsicológicas y psicoterapéuticas específicas que orientan de modo más o menos conscientesu desempeño profesional. El logro dela tarea impuesta por la epochéleexige, en palabras deYontef (1993), desarrollar una confianza plena en su propia experiencia inmediata y en la del paciente. Sin embargo, tal como yahemos señalado, la epochénunca puede pretender ser total. El encuentro terapéutico siempreestará circunscrito por las limitaciones que tanto el psicoterapeuta como el paciente experimentan en relación a su respectiva capacidad deconsciencia y habilidad para colocar entre paréntesissusparticulares sesgos personales y profesionales. Esta circunstancia, infranqueable desde el 
punto devista de lafenomenología, puede ser entendida como un obstáculo frustrante; no obstante, también puedeser comprendida como una realidad inevitableeinherenteacualquier proceso psicoterapéutico.

\section{El presente en el proceso psicoterapéutico}

Como yalo hemos mencionado, el método fenomenológico se basa en ladescripción de la experiencia inmediata tal como apareceen el presente. Esta perspectiva introduce una concepción específica en relación al tiempo en el marco dela psicoterapia humanista-existencial, la que a menudo ha sido caricaturizada al definirla como únicamente centrada en el "aquí-y-ahora" en desmedro del pasado y del futuro. En efecto, los psicoterapeutas deorientación humanista-existencial han enfatizado en repetidasocasioneslaimportancia del momento presentecomo ejeprimario dela existenciahumanay del proceso terapéutico (Brazier, 1993; Peñarrubia, 1998; Yontef, 1993). $\mathrm{N}$ o obstante, esteénfasis no pretende desval orizar la relevancia del pasado y del futuro en términos dela vivencia queel clientetienedesí mismo y desu situación vital. M ás bien, losterapeutas humanista-existenciales subrayan quetanto el recuerdo del pasado como la anticipación del futuro son fenómenos psicológicosque, invariablemente, se producen en el presente. La psicoterapia humanista-existencial reconocey asumela necesidad dequeel cliente sea capaz de comprender su propia historia y de construir un proyecto vital que lo guíe y que confiera sentido a su existencia. Sin embargo, destaca queel pacientevive, dehecho, en el presente, y queun proceso psicoterapéutico exitoso requierequese preste atención a esta circunstancia convirtiéndola en una dimensión central delasintervencionesclínicas.

Con todo, uno delosobjetivos cardinales dela psicoterapia humanista-existencial es el enriquecimiento y la profundización de la experiencia del cliente -en este sentido, el desarrollo de su capacidad para poner entre paréntesis sus propias preconcepciones y los propios prejuicios para acercarsea su experiencia de forma más abierta, contribuyen a promover y facilitar el cambio desu personalidad.

\section{Palabras finales}

Tal como indicamos al inicio de este artículo, en términos generales se ha asumido quela psicoterapia humanista-existencial sefundamenta fil osófica y epistemológicamente en la fenomenología y en el método fenomenológico, lo cual tiene diversas implicancias para la práctica clínica. Esta apreciación es ciertamentecorrecta; no obstante, la real idad psicoterapéutica es, sin lugar a dudas, más compleja y multifa- 
cética quela imagen quedeella ofrececual quier sistemateórico específico. A la luz delas múltiples exigencias clínicas y contextuales queenfrenta, un psicoterapeuta deorientación humanista-existencial no puedeser un fenomenólogo puro. A nuestro juicio, la actitud y el método fenomenológico quehemos descrito constituyen unabaseo plataforma para el accionar terapéutico en el marco de esta aproximación. El trabajo psicoterapéutico separece, sin embargo, a un ir y venir entrela apertura fenomenológicaa la experiencia presente y su comprensión conceptual a partir del conjunto de conocimientosy conceptosqueel clínico maneja como partedesu marco teórico de referencia.

La fenomenología por sí sola, dado su énfasis en la experienciadirectaeinmediata, no puededar cuenta (tampoco pretendehacerlo) deaspectos psicológicosclínicamenterelevantestalescomo el desarrollo de la personalidad o la estructura decarácter del paciente. Estos aspectos involucran la noción de una duración continuada de ciertos procesos subjetivos e intersubjetivos en el tiempo, un elemento del cual la fenomenologíacarece. Así, lafacilitación deprocesosterapéuticosimplica por partedel psicoterapeuta humanista-existencial la capacidad de fluctuar, de modo flexible, entre la conceptualización desu quehacer mediante sus teorías y la puesta entre paréntesis de ellasmismas. M artin Buber (1923) fue, tal vez, quien mejor graficó estacircunstancia: el ser humano serelaciona con el mundo y con los demás en dos formas complementarias y ambas son imprescindibles. Por un lado, el individuo sólo deviene verdaderamentehumano en cuanto serelaciona, demanera personal y comprometida, deyo atú; por otro, las exigencias prácticas de la vida leimponen la necesidad de establecer una relación impersonal y funcional con lascosas, deyo aello. D emodo similar, un trabajo psicoterapéutico óptimo incluyetanto una comprensión fenomenológica directadela experiencia del cliente, como un entendimiento conceptual deésta que está relativamenteal ejado de la inmediatez dela realidad vivencial del cliente. 


\section{Bibliografía}

Brazier, D . (1992). Phenomenological counseling and psychotherapy [on-line]. Amidatrust (www. amidatrust.com/article_phenomenological.html).

Brazier, D . (1993). Key concepts of phenomenological therapy [on-line]. Amidatrust (www.amidatrust.com/article_twelve.html).

Buber, M . (1923). Yo y tú. Buenos Aires: N ueva Visión.

Fran KL, V. (1946). Psicoanálisis y existencialismo: D ela psicoterapia a la logoterapia. M éxico: Fondo de Cultura Económica.

Genduin, E. (1966). Existencialismo y psicoterapia experiencial. En C. Alemany (Ed.), Psicoterapia experiencial y focusing: La aportación deE. T. Gendlin (pp. 31-56). Bilbao: D esclée de Brouwer.

Gen d LIn, E. (1973). Psicoterapia experiencial. En C. Alemany (Ed.), Psicoterapia experiencial y focusing: La aportación deE. T. Gendlin (pp. 143-195). Bilbao: D esclée de Brouwer.

Goldberg, A. (2001). Postmodern psychoanalysis [on-line]. Psyche M atters (http:// psychematters.com/papers/goldberg.htm).

Gold BERG, C. (2000). The metapsychological and philosophical dilemmas of psychoanalysis. International Journal of Psychotherapy, 5 (2), 133-144.

Halling, S. \& CARroll, A. (1999). Existential-phenomenological psychology. En D. M oss (Ed.), H umanistic and Transpersonal Psychology: A H istorical and Biographical Sourcebook (pp. 95-124). Westport: Greenwood Press.

H EGEL, G. (1807). Fenomenología del espíritu. M éxico: Fondo de Cultura Económica.

KRIZ, J. (1985). Corrientesfundamentalesen psicoterapia. Buenos Aires: Amorrortu.

M ARTín EZ, M . (1982). La psicología humanista: Fundamentación epistemológica, estructura y método. M éxico: Editorial Trillas.

M AY, R. (Ed.) (1961). Psicología existencial. Buenos Aires: Paidós.

M AY, R. (1969). Relation of existential to humanistic psychology. En A. Sutich \& M . Vich (Eds.), Readings in H umanistic Psychology (pp. 236-238). N ew York: The Free Press.

M o REIRA, V. (2001). M ásallá dela persona: H acia una psicoterapia fenomenológica mundana. Santiago de C hile: Editorial U niversidad de Santiago.

M oreira, V., Saboia, A., Beco, L. \& Soares, S. (1995). Psicoterapia fenomenológicoexistencial: Aspectos teóricos de la práctica clínica con base en las competencias. P sykhe, 4 (2), 121-129.

M OREN O, C. (2000a). Fenomenol ogía y filosofía exi stencial Volumen I: Enclavesfundamentales. M adrid: Síntesis.

M OREN O, C. (2000b). Fenomenología y filosofía existencial Volumen II: Entusiasmosy disidencias. M adrid: Síntesis.

M oss, D . (Ed.) (1999). H umanistic and Transpersonal Psychology: A H istorical and Biographical Sourcebook. Westport: Greenwood Press.

M oss, D . (2001). The roots and geneology of humanistic psychology. En K. Schneider, J. Bugental \& J. Fraser (Eds.), The H andbook of H umanistic Psychology: Leading Edges in Theory, Research, and Practice (pp. 5-20). California: Sage Publications.

Orange, D ., Atwood, G. \& Stolorow, R. (1997). Intersubjektivität in der Psychoanalyse: Kontextualismusin der psychoanalyti schen Praxis. Frankfurt am M ain: Brandes \& Apsel.

Peñ arrubia, F. (1998). Terapia G estalt: La vía del vací o fértil. M adrid: Alianza.

PerLs, F. (1947). Yo, hambrey agresión. M éxico: Fondo de Cultura Económica. 
PerLs, F., Goodman, P. \& H efferline, R. (1951). Terapia Gestalt: Excitación y crecimiento dela personalidad humana. M adrid: Los Libros del CTP.

Q uitm An N, H . (1985). Psicología humanística: C onceptosfundamentales y trasfondo fil osófico. Barcelona: H erder.

REICH , W. (1942). TheFunction of the O rgasm: Sex-Economic Problems of Biological Energy. London: Souvenir Press.

ReICH, W. (1949). Análisis del carácter. Barcelona: Paidós.

Rogers, C. (1951). Client-Centered Therapy: Its Current Practice, Implications, and Theory. Boston: H oughton M ifflin Company.

Rogers, C. (1961). El proceso deconvertirse en persona. Barcelona: Paidós.

So fF, M ., Ruh, M .\& Zabransky, D. (2004). Gestalttheorieund Feldtheorie. En M. H ochgerner, H. H offmann-W idhalm, L. N ausner \& E. W ildberger (Eds.), Gestalttherapie (pp. 13-36). Wien: Facultas.

Spagnu olo, M. (Ed.) (2001). Psicoterapia de la G estalt: H ermenéutica y clínica. Barcelona: Gedisa.

Sto lo Row, R., Atwood, G \& \& O range, D . (2002). Worlds of Experience: Interweaving Philosophical and Clinical Dimensions in Psychoanalysis. N ew York: Basic Books.

Stolorow, R., O range, D. \& Atwood, G. (1998). Thinking and working contextually: Toward a philosophy of psychoanalytic practice [on-line]. Self Psychology Page (http:// www.selfpsychology.org/neutrality/thinking.htm).

TAgEson, C. (1982). H umanistic Psychology: A Synthesis. Illinois: The D orsey Press.

T OTton, N . (2003). Body Psychotherapy: An Introduction. London: O pen U niversity Press.

V ELASCO, C. (2003). La psicología del self: U n abordaje epistemológico. Buenos Aires: Educa.

W ALdENFELs, B. (1992). D e H usserl a D errida: Introducción a la fenomenología. Barcelona: Paidós.

W OLF, E. (1988). Theorie und Praxis der psychoanalytischen Selbstpsychologie. Frankfurt am $M$ ain: Suhrkamp.

Yontef, G. (1993). Proceso y diálogo en psicoterapia gestáltica. Santiago de Chile: Cuatro Vientos.

Yontef, G. (1998). Preface to the G erman edition of Awareness, D ialogue, and Process [online]. Pacific G estalt Institute (www.gestalttherapy.org/ PREFACE3.htm).

Yontef, G. (2004). Zum Aspekt der Beziehung in Theorie und Praxis der Gestalttherapie [on-line]. Gestaltkritik, 1 (http://www.gestaltkritik.de/yontef_dialog.html). 